\title{
Dynamic Control of Radiative Surface Properties with Origami-Inspired Design
}

\author{
Rydge B. Mulford, Matthew R. Jones and Brian D. Iverson \\ Department of Mechanical Engineering \\ Brigham Young University
}

\section{ABSTRACT}

Thermal management systems for space equipment commonly use static solutions that do not adapt to environmental changes. Dynamic control of radiative surface properties is one way to respond to environmental changes and to increase the capabilities of spacecraft thermal management systems. This paper documents an investigation of the extent to which origamiinspired surfaces may be used to control the apparent absorptivity of a reflective material. Models relating the apparent absorptivity of a radiation shield to time-dependent surface temperatures are presented. Results show that the apparent absorptivity increases with increasing fold density and indicate that origami-inspired designs may be used to control the apparent radiative properties of surfaces in thermal management systems.

\section{INTRODUCTION}

Dynamic modification of radiative surface properties is highly desirable when designing systems that operate in environments where radiation is the dominant mode of heat transfer and radiative heat loads vary significantly. Such is the case for spacecraft in geosynchronous orbit or the exterior surfaces of terrestrial structures. However, intrinsic surface radiative properties are static and therefore unable to adapt to changing thermal environments, resulting in less-than-ideal operation for a significant fraction of a component's lifetime. Dynamic control of radiative surface properties would provide the ability to adapt surface behavior to the changing radiative environment.

Variation in the radiative environment occurs in several terrestrial and extra-terrestrial applications. As an illustration of adaptive radiation control, consider a satellite in geosynchronous orbit [1]. Satellite surfaces often exhibit a radiative surface property spectral distribution ideal for minimizing the net rate of heat transfer to a satellite when solar irradiation is present [2]. However, these static surfaces transfer heat to deep space when the satellite is shaded by the earth from the sun, cooling the satellite to unacceptable temperatures. Therefore, heaters must be used to prevent the satellite temperature from decreasing to unacceptable levels. The ability to vary the radiative surface properties of these radiators would allow optimized performance for varying conditions and potentially eliminate the need for power-consuming thermal management solutions [3]. Additional applications that would benefit from dynamic surface behavior include architectural exterior surfaces [4], IR detection concealment [5] and solar energy applications [6, 7].

Various technologies have been investigated in order to vary surface radiative properties [8-10]. Specifically, the use of surface coatings and thin films [11], electrowetting [12] and electrostatic actuation [13] have been explored. Louvers have also been used for thermal control but macro versions are generally bulky and not suitable for small satellites [14]. Electrochromic surfaces are a promising technology that can vary their emissivity through a wide range by application of a small voltage[15]. These devices, however, require time to adjust to changing environments and exhibit wide fluctuation in their spectral emissivity[16]. Thermochromic materials exhibit a change in emissivity with surface temperature. For these materials, no electrical or mechanical actuation is required, however, the radiative surface properties are entirely dependent on the surface temperature making it difficult for use in 
thermal management [17]. Geometrical modifications have also been considered, including the incorporation of micro-column arrays to increase absorptive properties [18] and radiator plates with specialized fractal geometries to increase emissive properties [19].

One dynamic solution yet to be considered in the literature involves the use of origami-inspired, dynamically variable, V-groove cavities. Multiple reflections within a cavity lead to increased apparent absorption and emission relative to a smooth surface of the same material. Apparent absorptivity is the ratio of irradiation absorbed by a surface to the irradiation incident on the surface [20] and can differ from a surface's intrinsic absorptivity (absorptivity of a flat surface). This increase in apparent absorption and emission for high aspect ratio cavities has been termed the cavity effect [21]. Several cavity geometries have been investigated to quantify the extent of the cavity effect on radiative properties relative to a flat surface. Cylindrical, conical, spherical, rectangular and $\mathrm{V}$-groove cavities are among the surface topographies that have been studied [21, 22].

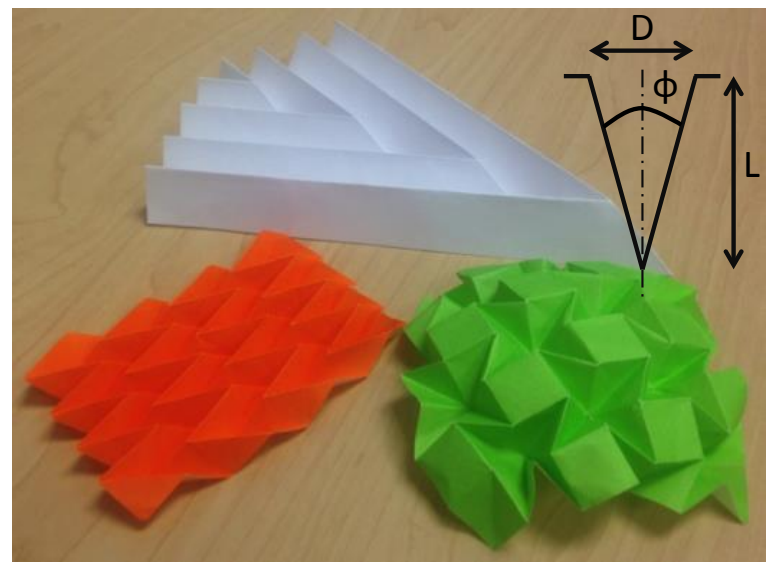

Figure 1. Sample origami structures with v-groove like cavities created from the folds. The inset indicates the v-groove cavity structure with varying cavity angle, $\phi$.

Consider the V-groove cavity shown in the inset of Figure 1. As the angle $(\phi)$ of the opening decreases, the aspect ratio $(L / D)$ increases and the apparent absorptivity and emissivity values increase [20-22]. The apparent radiative surface properties can approach those of a black surface when the surface is comprised of high aspect ratio cavities, independent of the intrinsic surface properties. Highly reflective surfaces transition from flat-surface behavior to black-like behavior at small cavity angles, while the transition occurs over a wider range of cavity angles for surfaces with lower intrinsic reflectivity.

Origami-based structures such as the miuraori and even simple accordion folds may be used to create a surface topography comprised of Vgroove cavities (see Figure 1). Origami has been shown to be an effective approach to controlling the motion of a compliant mechanism used for actuation and positioning $[23,24]$ with possible space applications including deployable solar arrays [25]. During deployment, origami structures transition from a folded to an expanded surface. A change in surface topography of this nature enables dynamic control of the apparent radiative surface properties through the cavity effect.

Origami-inspired, V-groove surfaces have the potential to significantly affect thermal control when radiative heat transfer is the dominant mechanism. Specifically, all possible absorptivity or emissivity values are obtainable between the material's intrinsic value and unity. Further, the desired absorptivity or emissivity condition can be achieved rapidly. Finally, surface degradation due to prolonged exposure may be accommodated simply by changing the fold density, which would extend the operating lifetime of a system. Active control of surface properties by topography manipulation enables the flexibility needed to respond to dynamic changes in the thermal environment or operating conditions.

This work demonstrates the range of variation in radiative surface properties for origami-inspired surfaces with linear actuation. First, a thermal model for determining the apparent absorptivity of a V-groove structure is presented. This model utilizes experimentally obtained temperatures and overall heat loss coefficients to calculate the apparent absorptivity. The experimental procedures to obtain the temperatures and heat loss coefficients are described and the data is presented for a flat surface and folded surfaces with five different cavity angles. This data is used in conjunction with the thermal model to calculate the apparent absorptivity for all six data sets (flat and folded). Calculated apparent absorptivity for flat surfaces is validated with measurements using 
an emissometer. Calculated apparent absorptivity for folded geometries is validated with $\mathrm{V}$-groove analysis by Sparrow [20].

\section{THERMAL MODELING}

Consider a surface that is uniformly irradiated by a blackbody source in quiescent air and isothermal surroundings. As illustrated in Figure 2 , the irradiated portion of the surface loses heat to the surroundings by convection and radiation and to the non-irradiated portion of the surface by conduction.

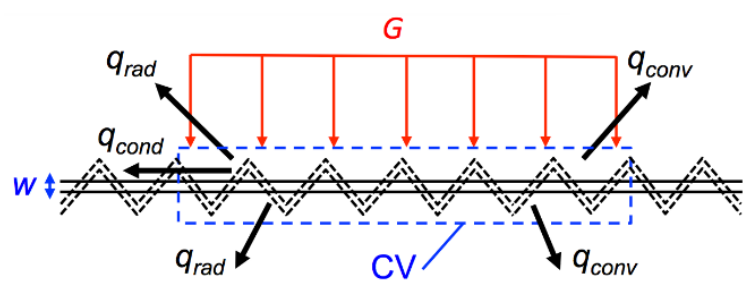

Figure 2. Schematic of the system used to model the relationship between the measured time-dependent, surface temperature profile and the absorptivity of the sample.

\section{General Approach}

Applying an energy balance for the system illustrated in Figure 2 gives Equation 1, where $\alpha_{a}$ is the apparent absorptivity, $G$ is the irradiation, $m=\rho A w$ is the mass in the control volume and $C$ is the specific heat. Since the surface is thin and has a high thermal conductivity, the illuminated portion of the surface is approximately isothermal. Assuming the radiative exchange with the surroundings may be linearized, the convective heat transfer coefficient is uniform, and conduction losses may be modeled using a shape factor, the heat loss terms may be grouped as shown in Equation 2.

$$
\begin{aligned}
& \alpha_{a} G_{B} A_{B}-\left(2 q_{\text {conv }}+2 q_{\text {rad }}+q_{\text {cond }}\right)=\rho A_{B} w C \frac{d T}{d t} \\
& q_{\text {loss }}(t)=\left(\frac{S k}{A}+2 h+2 h_{r}\right) A\left(T(t)-T_{\infty}\right)=U(t) A \cdot \theta(t)
\end{aligned}
$$

The Control Volume was assumed to be the same size for analysis of all fold densities. This allowed for elimination of $A_{B}$ from the analysis, simplifying the experimentation procedure. However, as the fold angle is increased, the amount of mass inside the control volume increases. This increased mass is accounted for by developing a ratio of the cross sectional area inside the control volume of a flat sample (as shown in Figure 5) to the cross sectional area inside the control volume of a folded sample. This ratio is given in Equation 3, where $\phi$ represents the opening angle of the $\mathrm{V}$ cavity. When added to the right side of Equation 1, this term compensates for the increased mass inside the control volume when in a folded state.

$$
\frac{A_{\text {unfolded }}}{A_{\text {folded }}}=\frac{1}{\sin \left(\frac{\phi}{2}\right)}
$$

This term was added to the right side of Equation 1. This allowed the governing differential equation for the temperature distribution of our system to be written as Equation 4.

$$
\frac{d \theta}{d t}+\left[\frac{\eta(\phi) U(t)}{U_{S S}}\right] \theta(t)=\frac{\alpha_{a}(\phi) G_{B} \eta(\phi)}{U_{S S}}
$$

Where:

$$
\eta(\phi)=\frac{U_{S S} \sin \left(\frac{\phi}{2}\right)}{\rho w C}
$$

\section{$U(t)$ Derivation}

To find a final value for apparent absorptivity, the relationship of $U$, the overall heat transfer coefficient, to time had to be determined. To do so, Equation 4 was modified to represent the same control volume as defined in Figure 2 but without the irradiative $G$ term, representing a foil at an elevated temperature cooling in ambient air.

The governing differential equation for $\theta$ during the cooling scenario is a homogeneous version of Equation 4. Since the time-dependent, temperature profile is measured during the cooling phase, the only unknown of Equation 4 when cooling is the overall heat transfer coefficient. Solving for $U$, we obtain: 


$$
U(t)=\frac{-\rho w C}{\sin \left(\frac{\phi}{2}\right)} \frac{1}{\theta} \frac{d \theta}{d t}
$$

The temperature data for a transiently cooled surface can be obtained by performing experiments with alternate heating (irradiation) and cooling to near ambient conditions. Further, $U$ obtained for a cooling surface can also be used as the overall heat transfer coefficient for an irradiated surface if $U$ can be expressed as a function of the temperature difference, $\Delta T$. The temperature difference of the cooling curve was plotted with respect to time, giving $\Delta T(t)$. This curve was then used to transform $U(t)$ into $U(\Delta T(t))$ such that an empirically obtained $U$ can be used in the calculation of the apparent absorptivity when the surface is irradiated. This allowed the irradiated $U_{S S}$ value to be evaluated from transient cooling temperature data.

With an empirically obtained overall heat transfer coefficient, the absorptivity can be now determined through a steady state method and two separate inverse approaches (based on Equation 4), outlined as follows.

\section{Steady State Method}

Steady state conditions were considered to determine an initial value for absorptivity. At steady state, Equation 4 simplifies to

$$
\alpha_{a}=\frac{U_{S S} \theta_{S S}}{G_{B}}
$$

Where $U_{S S}$ is the overall heat transfer coefficient at steady state and $\Theta_{S S}$ is the temperature difference at steady state. This method allows for calculation of a constant absorptivity that is independent of time, unlike the other two approaches. This method still relies on material properties and V-groove opening angle through the $\mathrm{U}_{\mathrm{SS}}$ calculation, as seen in Equation 6.

\section{Inverse Model 1: Direct Method}

Equation 4 was rearranged to yield absorptivity as a function of material properties, irradiation, and temperature data at each instant in time.

$$
\alpha_{a}(t)=\frac{U_{S S}}{G \eta(\phi)} \frac{d \theta}{d t}+\frac{U(t)}{G} \theta(t)
$$

Based on $U(\Delta T(t))$ obtained from the cooling curves for the same material and test case, the absorptivity was calculated by evaluating the derivative directly from a curve fit of the experimental temperature data.

\section{Inverse Model 2: Integrating Factor Method}

Returning to Equation 4, this first order, nonhomogeneous differential equation was solved using an integrating factor. For this approach, it was necessary to model $\mathrm{U}(\mathrm{t})$, as given in Equation 9 , in order to integrate the integrating factor.

$$
U(t)=\left(U_{S S}-U_{o}\right)\left[1-\exp \left(\frac{-t}{\tau}\right)\right]+U_{o}
$$

Here, $U_{S S}$ is the steady-state overall heat transfer coefficient, taken to be the maximum $U$ during a given heating cycle. The integrating factor is then given as

$$
\mu=\exp \left(\eta \tau \exp \left[\frac{-t}{\tau}\left(1-\frac{U_{o}}{U_{S S}}\right)\right]\right) \exp (\eta t)
$$

To determine $\tau, U(t)$ for multiple heating curves was computed using the method outlined for Equation 9. A curve was then fit to this data that followed the form of Equation 9. This curve provided $\tau$ for each set of data. Examining the range of $\tau$ values, it can be shown that the first exponential term in Equation 10 varies between 1.16 and 1.0. By approximating this term as 1.0, the integrating factor was simplified considerably. Applying this integrating factor to both sides of Equation 4 and integrating over $t$ allows us to find the final expression for apparent absorptivity, as given in Equation 11.

$$
\alpha_{a}(t)=\frac{\frac{U_{S S}}{G_{B}}\left[\theta-\exp (\eta(\phi) t) \theta_{o}\right]}{1-\exp (-\eta(\phi) t)}
$$


The final equations provided by the three approaches are given in Equations 7, 8 and 11. As can be seen, all three equations require the irradiative flux $\left(\mathrm{G}_{\mathrm{B}}\right)$, the overall heat loss coefficient $(\mathrm{U}(\mathrm{t}))$ and knowledge of a temperature profile during a heating scenario. Likewise, to evaluate $\mathrm{U}(\mathrm{t})$, a temperature curve for a cooling scenario must be obtained. Given these conditions, experimentation was necessary.

\section{EXPERIMENTAL SETUP}

The following section outlines the experimental conditions for transient temperature and flux measurements. An uncertainty analysis was also performed on the absorptivity models using the uncertainty values associated with measurements performed in this section.

\section{Transient Temperature Measurement}

Change in the apparent radiative properties of a surface was demonstrated through experimentation with a folded thin-foil heated by a blackbody cavity. A sheet of aluminum shim stock (alloy 1145) of thickness $25.4 \mu \mathrm{m}$ was folded into an accordion pattern. The folded sample was constrained within a test fixture that allowed the fold density to be varied from 0 (flat) to a Vgroove angle (See Figure 4a) of approximately $11^{\circ}$ without removing the sample from the fixture. The center of the sample was positioned concentric with the opening of a blackbody source (Land $\mathrm{R} 1200 \mathrm{P}), 15.4 \mathrm{~cm}$ away from the aperture. Two Ktype thermocouples (30 gauge) were attached to one fold (see Figure 3a) on the backside of the sample by means of thermal epoxy (Duralco 132). These thermocouples were placed immediately adjacent to the two folds and midway between the two peaks, all at the same vertical location. A third thermocouple monitored the room temperature throughout the testing.

The sample was alternately exposed to and then shielded from irradiation from the cavity by opening and closing a shutter. The blackbody emitter, set to $1000{ }^{\circ} \mathrm{C}$, was allowed to reach steady state operation before beginning the heating and cooling cycles. A two-color pyrometer (Omegascope OS3750) was used to confirm the temperature of the blackbody cavity. At a cavity set point of $1000{ }^{\circ} \mathrm{C}$, the pyrometer indicated a cavity temperature of $1000 \pm 1^{\circ} \mathrm{C}$. A piston linear actuator (BIMBA 093-RP) was positioned horizontally to the left of the blackbody aperture with a $6.4 \mathrm{~mm}$ thick insulated shutter attached directly in front of the aperture (Figure $3 b$ ). This insulated shutter acted to shield the blackbody radiation during cooling cycles. A LABVIEW program was used to control the piston linear actuator through use of an NI 9481 SPST digital output module attached to a power supply at $15 \mathrm{~V}$. When activated, this digital output module actuated a 5 port (SMC VF3320), solenoidactuated air valve, pressurizing the piston cylinder and causing the shutter to open and the sample to be irradiated. When deactivated, the cylinder was depressurized and the shutter was moved back into place by the spring-loaded air piston. Thermocouple readings (sampled at $3 \mathrm{~Hz}$ ) were also recorded using LABVIEW software. The shutter was opened for $80 \mathrm{~s}$ during the heating phase and closed for $100 \mathrm{~s}$ during the cooling phase. Thermal cycling was performed for approximately one hour with thermocouple data being collected continuously. Response time for the actuation was less than $0.5 \mathrm{~s}$ to open and close the shutter or less than $0.75 \%$ of the heating or cooling cycle times.

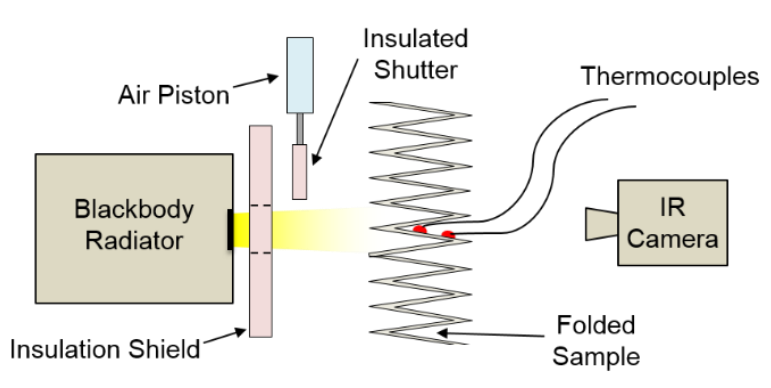

Figure 3. Schematic of the test configuration and temperature measurement for heating of the thin-foils using a blackbody cavity.

\section{Flux Measurement}

A Vatell HFM-7E/H heat flux gauge was mounted in a custom housing and attached to a three-axis optical rail system for positioning. The heat flux gauge was placed $15.4 \mathrm{~cm}$ away from the aperture of the blackbody cavity. The gauge was moved in $5 \mathrm{~mm}$ increments ( $\mathrm{X}$ and $\mathrm{Y}$ ) in the plane parallel to the front plane of the blackbody. Data over a circular irradiated area of $A_{B}=0.002 \mathrm{~m}^{2}$ (radius $=2.5 \mathrm{~cm}$ ) was averaged to determine the 
irradiation value for $\mathrm{G}$ used in the absorptivity calculations.

To confirm the values obtained from the heat flux gauge, a radiative heat transfer model was developed to calculate the irradiation on the sample $15.4 \mathrm{~cm}$ away from the blackbody. Equation 3 was used to calculate the irradiation incident on the heat flux gauge, where $F_{\text {Gauge } \rightarrow \text { Blackbody }}$ is the view factor of the blackbody cavity as seen by the gauge and obtained for two concentric circles of known radii at a specified distance.

$$
G_{B}=\sigma T_{B}^{4} F_{\text {Gauge } \rightarrow \text { Blackbody }}
$$

This model assumed blackbody behavior in a non-participating media with a cavity temperature of $1000{ }^{\circ} \mathrm{C}$. Measured flux values at a distance of $15.4 \mathrm{~cm}$ are compared in the results section.

\section{$\underline{\text { Uncertainty }}$}

Least count uncertainty values for measured values and experimental parameters are provided in Table 1. These uncertainties are utilized in an uncertainty analysis performed on calculated absorptivity values in the results section to follow.

Table 1. Least count uncertainty values for each measurement/parameter used in absorptivity calculations.

Table 1. Least count uncertainty values for the various measured parameters used in experimentation.

\begin{tabular}{ccc} 
Parameter & Least Count Uncertainty & Unit \\
\hline \hline$G$ & 31.5 & $\mathrm{~W} / \mathrm{m}^{2}$ \\
$C_{p}$ & 4.5 & $\mathrm{~J} /(\mathrm{kg} \mathrm{K})$ \\
$\rho$ & 6.8 & $\mathrm{~kg} / \mathrm{m}^{3}$ \\
$\theta$ & 1.5 & $\mathrm{~K}$ \\
$w$ & 1.27 & $\mu \mathrm{m}$ \\
$t$ & 10 & $\mu \mathrm{s}$ \\
\hline
\end{tabular}

\section{RESULTS}

\section{Temperature Curves}

Figure 4 illustrates the time-dependent temperatures for the thermocouples attached to the backside of a folded sample for a flat sample and a sample with $\phi=14^{\circ}$ during cyclic heating and cooling. During experimentation, the heating/cooling cycles were repeated until quasisteady behavior was observed, as shown by the vertical black line in Figure 4. Note the consistency of the peak temperature after heating and the minimum temperature after cooling for fixed periods of time. MATLAB was used to fit a curve to the 8 temperature curves on the right side of the vertical black line in Figure 4, giving a time averaged heating and cooling curve for use in determining both $\mathrm{U}(\mathrm{t})$ and apparent absorptivity.

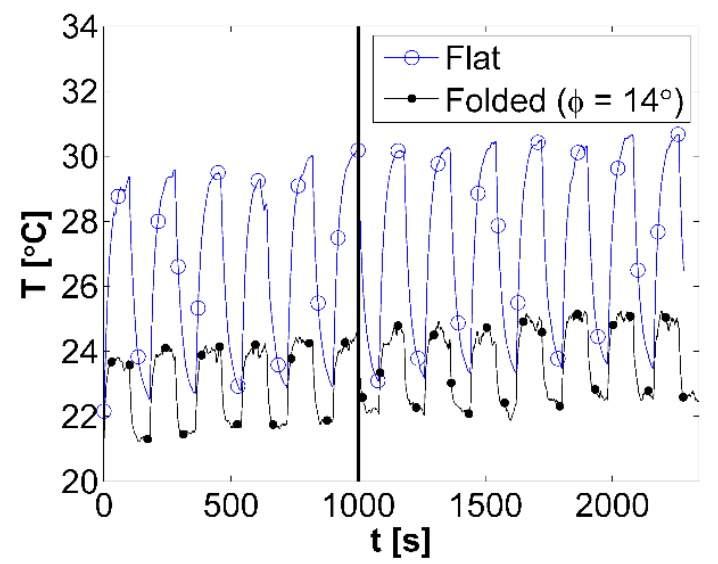

Figure 4. Heating and cooling curves averaged over 8 cycles for surfaces that range in cavity angle from a flat surface to a surface with $\phi \cong 14^{\circ}(\mathrm{L} / \mathrm{D} \cong 4)$, indicating the increase in surface temperature for the same heating condition resulting from an increased apparent absorptivity with reducing cavity angle.

\section{Heat Flux}

Heat flux measurements are illustrated in Figure 6 along a vertical and horizontal line with the intersection of these lines corresponding to the axis of the blackbody cavity. Measurements were taken at $5 \mathrm{~mm}$ increments over the entire $25 \mathrm{~mm}$ radius around the blackbody axis and averaged to obtain a flux value of $950 \mathrm{~W} / \mathrm{m}^{2}$. 


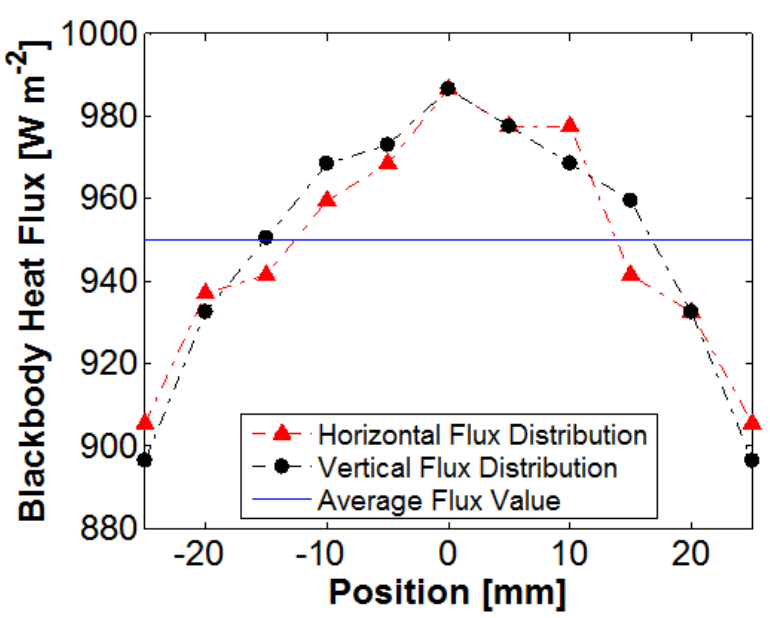

Figure 5. Horizontal and vertical flux distributions measured over a $25 \mathrm{~mm}$ radius centered about the blackbody cavity axis. The average value over this region $\left(950 \mathrm{~W} / \mathrm{m}^{2}\right)$ is also plotted.

\section{Flat Sample Apparent Absorptivity}

With temperature and time data collected for a flat sample, the inverse model can be used to predict the apparent absorptivity of a sample. Using Equation 6, the overall heat transfer coefficient of the flat sample was computed and is plotted in Figure 7a. This U(t) curve was then used in conjuction with Equations 7, 8 and 11 to derive the apparent absorptivity of the flat sample. These results are given in Figure $7 \mathrm{~b}$. The Steady State method is a constant value of 0.028 and the Direct method varies little from this value. The Integrating Factor method begins at a higher value but converges to the Steady State method value. As such, the absorptivity of the flat surface was determined to be 0.028 .

An uncertainty analysis was performed on the three absorptivity calculation methods using the least count uncertainty values given in Table 1. The root sum square of the partial derivative multiplied by the uncertainty of each parameter was used to calculate the total uncertainty for absorptivity. Uncertainty in the calculated absorptivity is plotted with the results in Figure $6 b$.

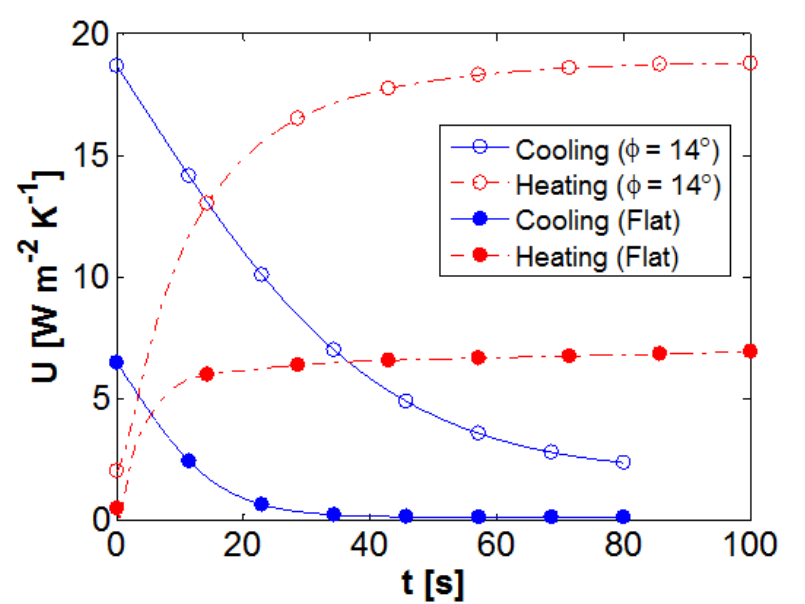

(a)

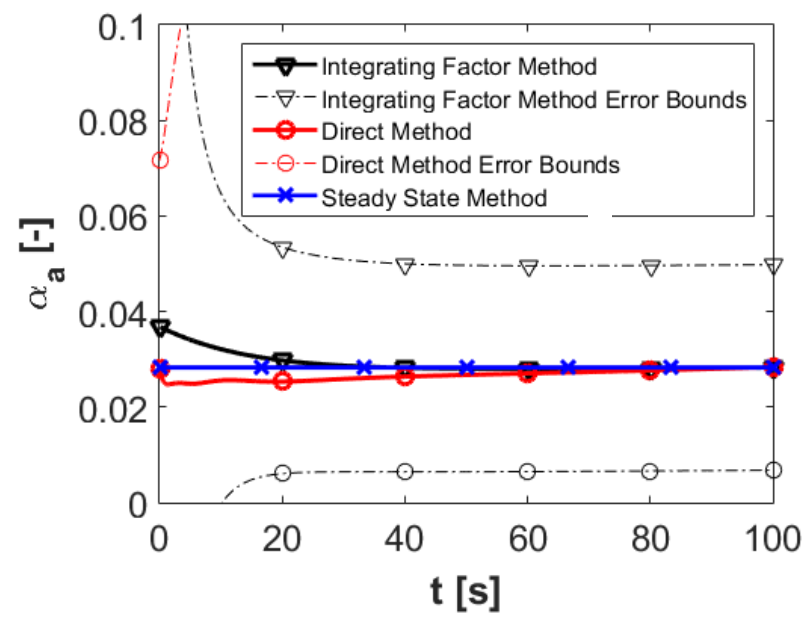

(b)

Figure 6. (a) Computed $U$ value for heating and cooling portions of the cyclic heating on a flat sample and a sample with $\phi=11^{\circ}$. (b) Computed absorptivity values for the direct and integrating factor inverse methods.

In an effort to validate the flat surface inverse model for absorptivity, a flat surface of the aluminum shim stock used in the testing described above was analyzed with an ET-100 Emissometer. An integrating sphere collected all reflected radiation from the surface of the test surface when irradiated at a near-normal angle. The reflectance measurements were recorded over six discrete wavelength bands in the infrared region and then weighted against the spectral distribution of the incoming irradiation. Using this method, the total, hemispherical absorptivity was calculated as 0.028 \pm 0.001 (an average of three measurement tests). These results correlate very well with the results from the thermal model, validating the approach. 


\section{Folded Sample Apparent Absorptivity}

The three approaches used to calculate the apparent absorptivity of a flat surface were used to also find the apparent absorptivity of folded surfaces. Figure 4 shows sample temperature data obtained for a folded surface with $\phi_{4}=14^{\circ}$ and the $U(\Delta T(t))$ curve as calculated from this temperature data is given in Figure 7a. Using this information, the Steady State method, Direct method and Integrating Factor method approaches yielded apparent absorptivity results as presented in Figure 9. The results show an increase in apparent absorptivity from 0.028 for the flat surface to 0.21 for the folded surface with $\phi_{4}=14^{\circ}$. This represents an increase by almost one order of magnitude with smaller cavity angles resulting in even greater increases for apparent absorptivity. The Steady State results and the Direct method results correlate well; the Integrating Factor method begins with an initial offset and converges to the steady state value. As fold density increases, the apparent absorptivity of the surface likewise increases. The direct method, steady state method and integrating factor method produce similar results for all fold densities.

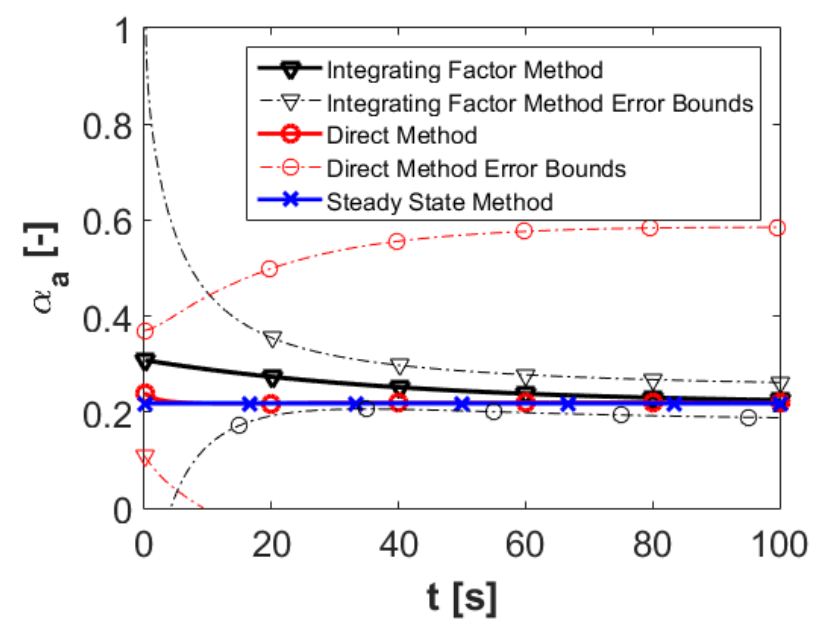

Figure 8. The apparent absorptivity of a folded sample $\left(\phi=14^{\circ}\right)$ as given by the three approaches. The results all converge to one common value, 0.21 .

To validate the thermal model, the experimental results were compared with the work of Sparrow [20]. Sparrow solved for the apparent absorptivity of a V-groove using a ray counting technique independent of experimental measurements completely unrelated to our approach. As such, any correlation between the thermal model approach and Sparrow's approach would validate the thermal model. As seen in Figure 9, the results of the two models correlate well, with the thermal model results falling within error range of Sparrow's results at all tested fold angles.

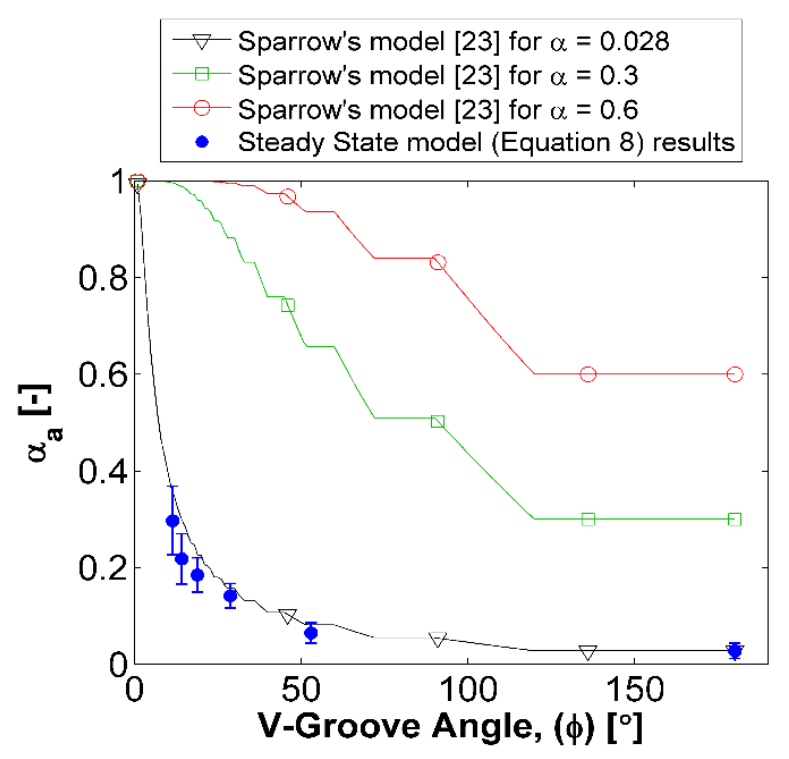

Figure 9. The apparent absorptivity of a surface as a function of V-groove Angle. Solid lines depict Sparrow's [20] results while the blue dots depict the results of our thermal model. This allows for a comparison of theoretical and experimental methods.

\section{CONCLUSIONS}

Thermal modeling and experimentation have shown that the apparent absorptivity of an origami-inspired, V-grooved, surface increases with decreasing cavity angle. Surface temperatures exhibit higher maximum temperatures for the same incident heat flux and heating time when the fold density is high, corresponding to a higher apparent absorptivity due to the cavity effect. The inverse models developed here correctly predict the apparent absorptivity as a function of opening angle, surface properties and irradiation and have been benchmarked with flat surface emissometer measurements and classical V-groove analytical models. 


\section{FUTURE WORK}

Future work includes investigating alternative surface materials, more elaborate origami folding patterns and the development of methods to examine diffuse, non-conductive materials. Total surface emission and the tradeoff associated with a fixed amount of material is also being explored.

\section{ACKNOWLEDGMENTS}

This work was funded through a grant from the Experimental Program to Stimulate Competitive Research from NASA through the Rocky Mountain Space Grant Consortium.

\section{REFERENCES}

[1] Mulford, R. B., Christensen, L. G., Jones, M. R., and Iverson, B. D., 2014, "Dynamic control of radiative surface properties with origami-inspired design," ASME International Mechanical Engineering Congress and Exposition, ASME, Montreal, Canada.

[2] Gilmore, D. G., 2002, "Spacecraft thermal control handbook," The Aerospace Corporation, El Segundo, CA.

[3] Grob, L. M., and Swanson, T. D., "Parametric study of variable emissivity radiator surfaces," Proc. Space Technology and Applications International Forum, M. S. El-Genck, ed., American Institute of Physics, pp. 809-814. [4] Akbari, H., and Konopacki, S., 2004, "Energy effects of heat-island reduction strategies in toronto, canada," Energy, 29(2), pp. 191-210. [5] Beaupre, S., Breton, A.-C., Dumas, J., and LeClerc, M., 2009, "Multicolored electrochromic cells based on poly derivatives for adaptive camouflage," Chemistry of Materials, 21, pp. 1504-1513.

[6] Li, M., Sun, C. J., Wang, R. Z., and Cai, W. D., 2004, "Development of no valve solar ice maker," Applied Thermal Engineering, 24(5-6), pp. 865-872.

[7] Anyanwu, E. E., and Ogueke, N. V., 2007, "Transient analysis and performance prediction of a solid adsorption solar refrigerator," Applied Thermal Engineering, 27(14-15), pp. 2514-2523. [8] Shannon, K. C., Sheets, J., Groger, H., Storm, R., and Williams, A., 2008, "Thermal management integration using plug and play variable emissivity devices," $22^{\text {nd }}$ Annual
AIAA/USU Conference on Small

SatellitesLogan, UT.

[9] Baturkin, V., 2005, "Micro-satellites thermal control-concepts and components," Acta Astronautica, 56(1-2), pp. 161-170.

[10] Hill, S. A., Kostyk, C., Moril, B., Notardonato, W., Rickman, S., and Swanson, T., 2012, "Thermal management systems roadmap, technology area 14," NASA, Washington, D.C. [11] Hale, J. S., DeVries, M., Dworak, B., and Woollam, J. A., 1998, "Visible and infrared optical constants of electrochromic materials for emissivity modulation applications," Thin Solid Films, 313-314(1-2), pp. 205-209.

[12] Gong, J., Cha, G., Ju, Y. S., and Kim, C.-J., 2008, "Thermal switches based on coplanar EWOD for satellite thermal control," IEEE International Conference on Micro Electro Mechanical SystemsTucson, AZ. [13] Biter, W., Hess, S., Oh, S., Douglas, D., and Swanson, T., 2005, "Electrostatic radiator for satellite temperature control," IEEE Aerospace ConferenceBig Sky, MT.

[14] Gilmore, D. G., 2002, "Spacecraft thermal control handbook," Aerospace Press, El Segundo, CA.

[15] Bergeron, B. V., White, K. C., Boehme, J. L., Gelb, A. H., and Joshi, P. B., 2008, "Variable absorptance and emittance devices for thermal control," Journal of Physical Chemistry, 112, pp. 832-838.

[16] Demiryont, H., and Moorehead, D., 2009, "Electrochromic emissivity modulator for spacecraft thermal management," Solar Energy Materials and Solar Cells, 93(12), pp. 2075-2078. [17] Benkahoul, M., Chaker, M., Margot, J., Haddad, E., Kruzelecky, R., Wong, B., Jamroz, W., and Poinas, P., 2011, "Thermochromic vo2 film deposited on al with tunable thermal emissivity for space applications," Solar Energy Materials and Solar Cells, 95(12), pp. 3504-3508. [18] Adjim, M., Pillai, R., Bensaoula, A., Starikov, D., Boney, C., and Saidane, A., 2007, "Thermal analysis of micro-column arrays for tailored temperature control in space," Journal of Heat Transfer, 129(7), p. 798.

[19] Dannelley, D., and Baker, J., 2013, "Radiant fin performance using fractal-like geometries," Journal of Heat Transfer, 135(8), p. 081902. [20] Sparrow, E. M., and Lin, S. H., 1962, "Absorption of thermal radiation in a $\mathrm{v}$-groove 
cavity," International Journal of Heat and Mass

Transfer, 5, pp. 1111-1115.

[21] Sparrow, E. M., and Cess, R. D., 1978,

Radiation heat transfer, McGraw-Hill, New York.

[22] Modest, M. F., 2013, Radiative Heat

Transfer, Academic Press, New York.

[23] Greenberg, H. C., Gong, M. L., Magleby, S.

P., and Howell, L. L., 2011, "Identifying links

between origami and compliant mechanisms,"

Mechanical Sciences, 2(2), pp. 217-225.

[24] Bowen, L. A., Grames, C. L., Magleby, S.

P., Howell, L. L., and Lang, R. J., 2013, "A
Classification of Action Origami as Systems of Spherical Mechanisms," Journal of Mechanical Design, 135(11), p. 111008.

[25] Zirbel, S. A., Lang, R. J., Thomson, M. W., Sigel, D. A., Walkemeyer, P. E., Trease, B. P., Magleby, S. P., and Howell, L. L., 2013, "Accommodating thickness in origami-based deployable arrays," Journal of Mechanical Design, 135(11), p. 111005. 